LA-UR-01-2673

Approved for public release; distribution is unlimited.

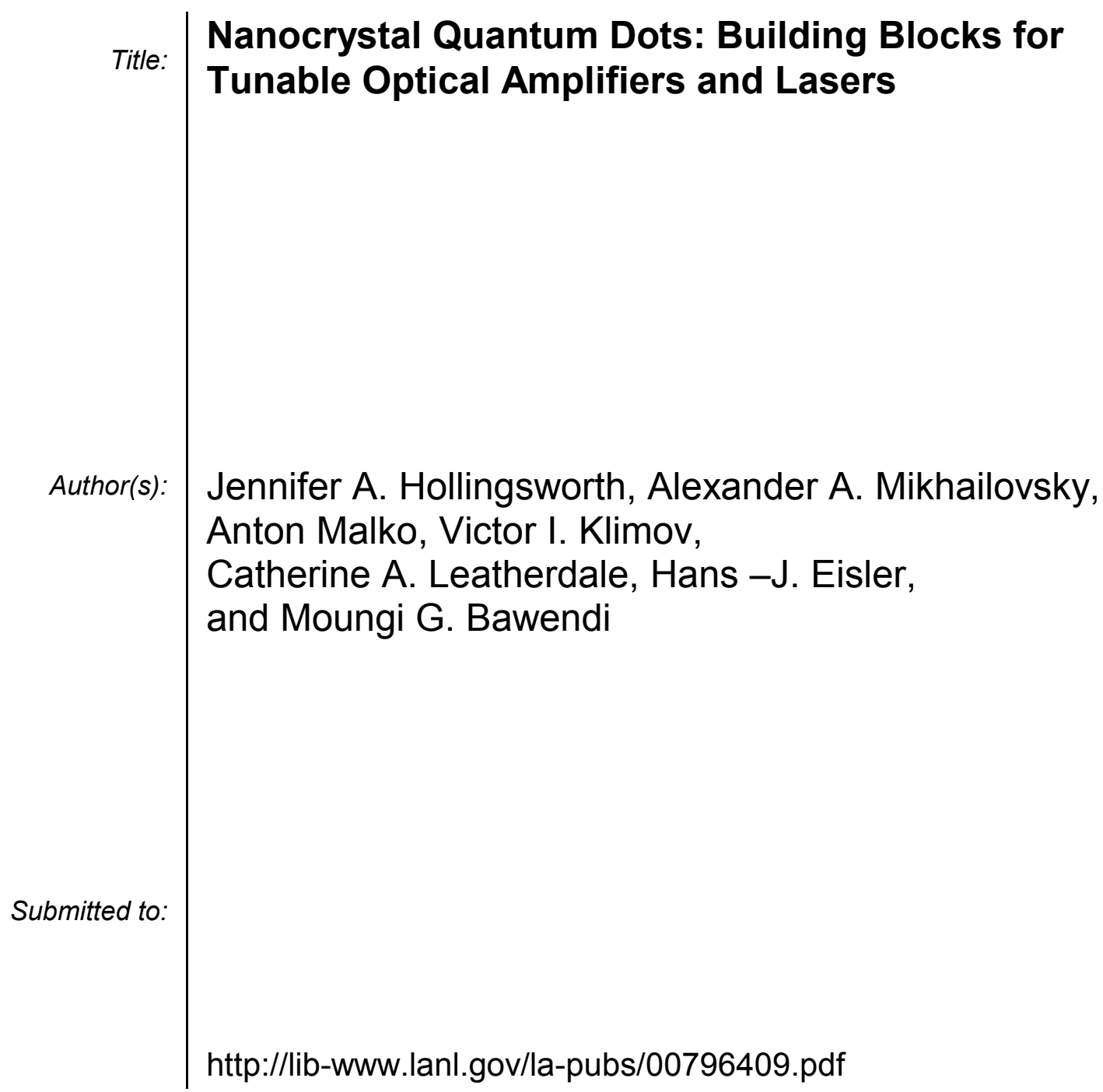

Los Alamos National Laboratory, an affirmative action/equal opportunity employer, is operated by the University of California for the U.S. Department of Energy under contract W-7405-ENG-36. By acceptance of this article, the publisher recognizes that the U.S. Government retains a nonexclusive, royaltyfree license to publish or reproduce the published form of this contribution, or to allow others to do so, for U.S. Government purposes. Los Alamos National Laboratory requests that the publisher identify this article as work performed under the auspices of the U.S. Department of Energy. Los Alamos National Laboratory strongly supports academic freedom and a researcher's right to publish; as an institution, however, the Laboratory does not endorse the viewpoint of a publication or guarantee its technical correctness. 


\title{
Nanocrystal Quantum Dots: Building Blocks for Tunable Optical Amplifiers and Lasers
}

${ }^{1}$ Jennifer A. Hollingsworth, ${ }^{1}$ Alexander A. Mikhailovsky, ${ }^{1}$ Anton Malko, ${ }^{1}$ Victor I. Klimov, ${ }^{2}$ Catherine A. Leatherdale, ${ }^{2}$ Hans -J. Eisler, and ${ }^{2}$ Moungi G. Bawendi,

${ }^{1}$ Physical Chemistry and Applied Spectroscopy, Chemistry Division, Los Alamos National Laboratory, Los Alamos, NM 87545, USA

${ }^{2}$ Department of Chemistry and Center for Materials Science and Engineering, Massachusetts Institute of Technology, Cambridge, MA 02139, USA

\begin{abstract}
We study optical processes relevant to optical amplification and lasing in CdSe nanocrystal quantum dots (NQD). NQDs are freestanding nanoparticles prepared using solution-based organometallic reactions originally developed for the $\mathrm{Cd}$ chalcogenides, CdS, CdSe and CdTe [J. Am. Chem. Soc. 115, 8706 (1993)]. We investigate NQDs with diameters ranging from 2 to $8 \mathrm{~nm}$. Due to strong quantum confinement, they exhibit sizedependent spectral tunability over an energy range as wide as several hundred meV. We observe a strong effect of the matrix/solvent on optical gain properties of CdSe NQDs. In most of the commonly used solvents (such as hexane and toluene), gain is suppressed due to strong photoinduced absorption associated with carriers trapped at solvent-related interface states. In contrast, matrix-free close packed NQD films (NQD solids) exhibit large optical gain with a magnitude that is sufficiently high for the optical gain to successfully compete with multiparticle Auger recombination [Science 287, 10117 (2000)]. These films exhibit narrowband stimulated emission at both cryogenic and room temperature, and the emission color is tunable with dot size [Science 290, 314 (2000)]. Moreover, the NQD films can be incorporated into microcavities of different geometries (micro-spheres, wires, tubes) that produce lasing in whispering gallery modes. The facile preparation, chemical flexibility and wide-range spectral tunability due to strong quantum confinement are the key advantages that should motivate research into NQD applications in optical amplifiers and lasers.
\end{abstract}

\section{INTRODUCTION}

It was realized almost two decades ago that semiconductor nanocrystal quantum dots (NQDs) should provide superior performance in lasing applications in comparison with other semiconductor bulk and low dimensional materials. In very small dots, the spacing of the electronic states is much greater than the available thermal energy (strong confinement), inhibiting thermal depopulation of the lowest electronic states. This effect should result in a lasing threshold that is temperature insensitive at an excitation level of only one electron-hole (e-h) pair per dot on average [1]. Additionally, NQDs in the strong confinement regime have an emission wavelength that is a pronounced function of size, adding the advantage of continuous spectral tunability over a wide energy range simply by changing the size of the dots. The prospect of realizing lasers for which the output 
color can be controlled by facile manipulation of the dot size and semiconductor composition has been a driving force in NQD research for more than a decade.

Lasing has previously been demonstrated for epitaxially grown III-V NQDs [2-4]. These dots have relatively large lateral sizes (typically greater than $10 \mathrm{~nm}$ ); therefore, the spacing between their electronic states is smaller than room-temperature carrier energies (weak confinement) and the lasing threshold is temperature sensitive. Further, large lateral dimensions and difficulties in size control limit their spectral tunability using quantum confinement effects. As a result, the emission wavelengths in epitaxial dots are usually controlled by a material's composition rather than QD size [4].

Direct colloidal chemical synthesis provides routine preparations of freestanding semiconductor nanoparticles with sizes that correspond to the regime of extremely strong confinement. For the studies reported here, we synthesized CdSe NQDs via the pyrolysis of dimethylcadmium $\left(\mathrm{CdMe}_{2}\right)$ and trioctylphosphine selenide (TOPSe) in a coordinating solvent mixture of trioctylphosphine (TOP) and trioctylphosphine oxide (TOPO), as schematically shown in figure 1 [5]. In addition to providing the medium for the solutionbased reactions, the coordinating solvent molecules covalently bind to the NQD surface atoms, forming an organic layer, or cap, on the surfaces of the individual NQDs. This capping provides electronic and chemical passivation of surface dangling bonds and imparts solubilities and reactivities that are characteristic of the organic ligand. Asprepared NQDs are further size selected using size-selective precipitation that allows isolation of ensembles with size dispersions of $<5 \%$. Particles of $\sim 2$ to $8 \mathrm{~nm}$ in diameter were prepared, spanning the range from strong quantum confinement to weak confinement.

In this size range electronic interlevel spacings can exceed hundreds of meV, and size-controlled spectral tunability over an energy range as wide as $1 \mathrm{eV}$ can be achieved. Furthermore, improved schemes of surface passivation allow significant suppression of surface trapping and produce room-temperature photoluminescence (PL) quantum efficiencies as high as $50 \%$ with emission wavelengths tunable across the entire visible spectrum. However, despite their excellent emission properties, favorable for optical amplification, colloidal nanostructures have failed to yield lasing in numerous efforts. In this paper we analyze the underlying physics of processes relevant to optical gain and lasing in strongly confined NQDs. We show that there are solvent/interface effects that prevent the observation of gain in most common NQD/solvent systems. Further, we show that there are intrinsic Auger nonradiative recombination processes that complicate the development of stimulated emission in strongly confined NQDs, but do not inherently prevent it. Finally, we demonstrate stimulated emission in close-packed films of CdSe nanocrystal NQDs in the strong confinement regime that provides for the first time proofof-principle for $N Q D$ lasing.

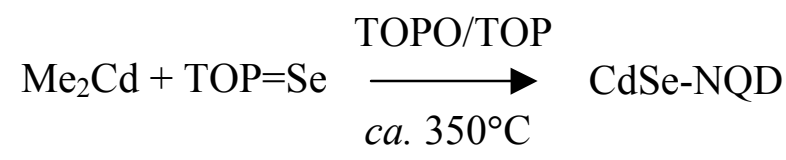

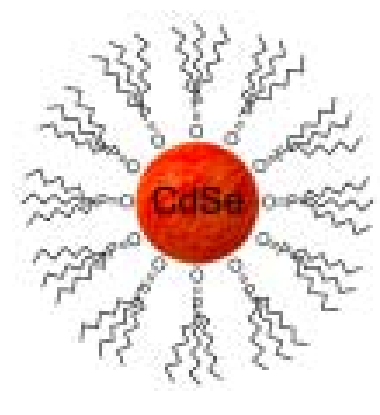

Figure 1. An organometallic reaction used to fabricate CdSe NQDs surface passivated with an organic capping layer; TOPO molecules of cap are shown. 


\section{EXPERIMENTAL DETAILS}

CdSe NQDs were fabricated as shown in figure 1 [5]. NQD solids were prepared by drop casting films from hexane/octane solutions; solvent evaporation yielded amorphous dry solid films of close-packed NQDs. Transient absorption (TA) measurements in the visible were performed using a femtosecond (fs) pump-probe experiment as previously described [6]. Briefly, the samples were pumped at $3.1 \mathrm{eV}$ by frequency doubled 100 fs pulses from an amplified Ti-sapphire laser. Pump-induced absorption changes were measured using time-delayed broad-band probe pulses of a fs white light continuum generated in a sapphire window.

\section{RESULTS/DISCUSSION}

\section{Solvent/interface dependent optical gain dynamics in CdSe NQDs}

In an attempt to observe gain in NQD CdSe solutions, we studied the TA pump dependence for NQD CdSe of various sizes in hexane (figure 2). The gain threshold corresponds to $-\Delta \alpha / \alpha_{0}=1$, where $\alpha_{0}$ is a linear absorption coefficient and $\Delta \alpha$ a pumpinduced absorption change. None of the samples in figure 2 shows crossover from absorption to gain even at very high pump densities corresponding to $\mathrm{N}_{\mathrm{eh}}>10$, where $\mathrm{N}_{\mathrm{eh}}$ is the number of electron-hole pairs per dot. Rather, a "universal" size-independent TA pump-intensity dependence, showing a saturation of the $1 \mathrm{~S}$ absorption bleaching before crossover to gain, is observed. The universal curve shows a linear growth below $\mathrm{N}_{\mathrm{eh}}=1$ and saturation at a level $-\Delta \alpha / \alpha_{0} \approx 1$ above $\mathrm{N}_{\text {eh }}=1$.

These data are compared with the pump dependence expected for state filling in a system for which the $1 \mathrm{~S}$ optical transition couples a populated electron state to either a populated (dashed line) or an unpopulated (solid line) hole state. The data are consistent with the latter, suggesting fast hole relaxation to a state that is lower in energy than that involved in the 1S transition [7]. We recently studied this relaxation process using an ultrafast PL experiment [8]. Fs PL data indicate a sub-ps hole relaxation from the state responsible for the $1 \mathrm{~S}$ absorption ("absorbing" state) to a lower energy state involved in

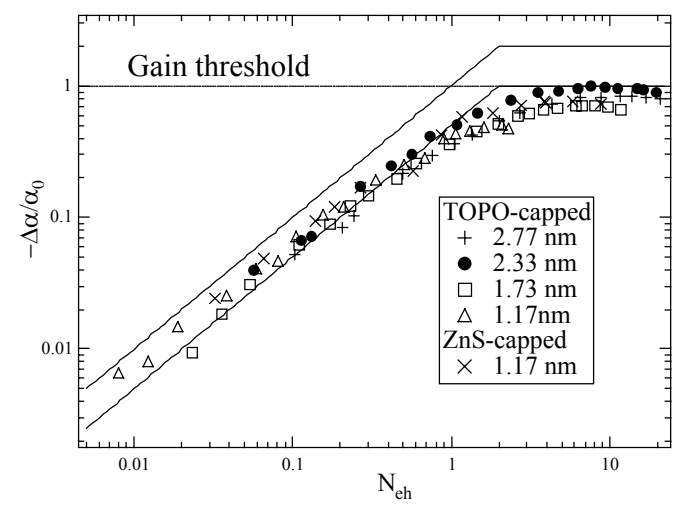

Figure 2. Pump dependence of the normalized $1 S$ absorption changes in a NQD-CdSe/hexane sample.

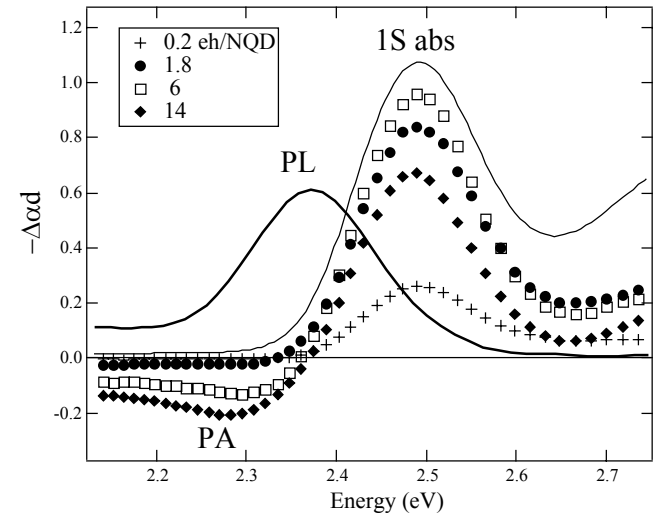

Figure 3. Pump dependence of $1 S$ bleaching $(\Delta \alpha<0)$ and photoinduced absorption $(\Delta \alpha>0)$ in a NQD-CdSe/hexane sample. 
the band-edge PL ("emitting" state). The formation of these "fine-structure" hole states is due to splitting of the lowest $1 \mathrm{~S}_{3 / 2}$ hole level induced by electron-hole exchange coupling, interactions with crystalline field and NQD shape asymmetry [9]. The transition involving the low-energy hole state is not well pronounced in absorption due to its relatively small oscillator strength, but since it is the lowest in energy it is responsible for processes involving light emission and, hence, optical gain.

Instead of gain, however, CdSe NQD/hexane samples show photoinduced absorption (PA) in the region of the lowest "emitting" transition (figure 3). In contrast to $1 \mathrm{~S}$ bleaching which saturates at high pump intensities, PA does not show saturation and, therefore, cannot be circumvented by simply increasing the excitation density. Analysis of TA data for NQDs in different liquid and solid-state matrices indicate that the PA is dependent on the matrix material, suggesting that it is due to excited-state absorption involving carriers trapped at solvent-related interface states. Such commonly used solvents as toluene, chloroform and HMN show a PA band comparable in intensity with that in hexane (figure 4). However, in the case of NQDs dispersed in polyvinyl butyral, the PA is reduced in magnitude and is red shifted with respect to the PL band. The strongest PA suppression was observed for TOP, one of the NQD growth solvents. CdSe NQDs/TOP samples show gain at the position the PL band (figure 5). The transition from absorption to gain occurred at carrier densities between 1 and 2 electron-hole (e-h) pairs per dot on average, consistent with the $1 \mathrm{e}-\mathrm{h}$ pair theoretical threshold expected for $3 \mathrm{D}$ strongly confined systems. The gain saturated at a level of $\sim 0.7 \alpha_{0}$, corresponding to $\sim 70 \%$ population inversion in terms of a simple two-level model.

Despite the fact that CdSe NQD/TOP samples show optical gain, they do not exhibit stimulated emission. In this case, as well as in other cases of relatively dilute NQD systems (e.g., NQD-doped glasses fabricated by high-temperature precipitation), the development of stimulated emission is inhibited by ultrafast decay of the optical gain, as analyzed below.

\section{Multiparticle Auger recombination and optical gain dynamics in NQDs}

In NQDs the band-edge optical gain is dominated by two e-h pair states, i.e. by a quantum-confined biexciton. As shown by our previous studies of multiparticle dynamics

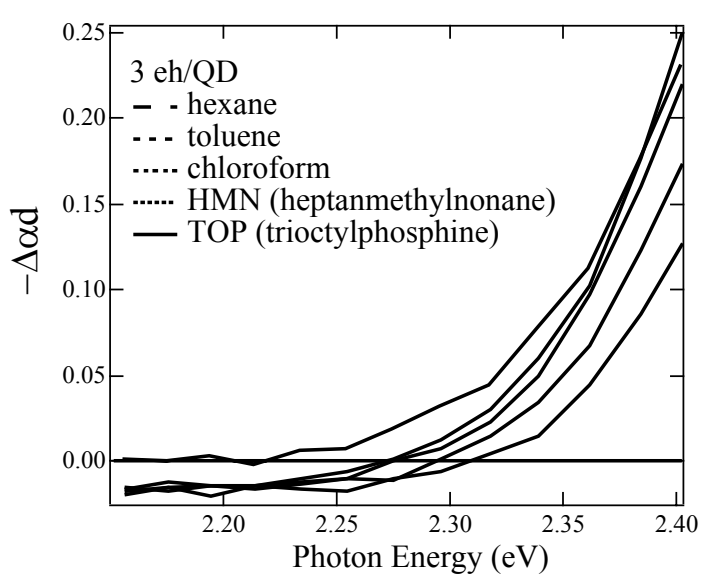

Figure 4. Solvent-dependent PA.

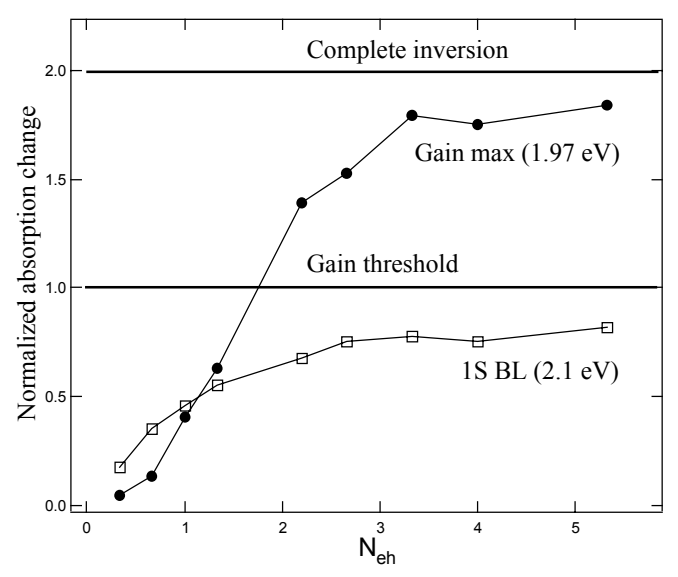

Figure 5. Gain observed in NQD-CdSe/TOP sample is red-shifted from the $1 \mathrm{~S}$ bleach band, where no gain is observed. 
[10], the biexciton decay in CdSe NQDs is dominated by Auger recombination. Auger recombination is a nonradiative process that leads to the recombination of e-h pairs via energy transfer to a third particle (an electron or a hole) that is re-excited to a higher energy state within the dot or even outside it (Auger ionization). Auger recombination has a relatively low efficiency in bulk semiconductors, for which significant thermal energies are required to activate the effect. However, Auger decay is greatly enhanced in quantum confined systems for which the relaxation in momentum conservation removes the activation barrier [11].

In figure 6 we show the size-dependence of the biexciton decay. These data indicate that the biexciton lifetime $\left(\tau_{2}\right)$ rapidly decreases with decreasing NQD size following a cubic size dependence $\left(\tau_{2} \propto R^{3}\right) ; \tau_{2}=363$ ps in NQDs with $R=4.1$ and reduces to only $6 \mathrm{ps}$ for $R=1.2 \mathrm{~nm}$. Due to its very short time constants, nonradiative Auger relaxation of doubly excited nanoparticles dominates over surface trapping even in samples with incomplete surface passivation. This implies that the fast optical gain decay due to Auger recombination is the principal factor inhibiting lasing in small-size colloidal dots.

\section{Stimulated emission in NQDs: Proof of principle for NQD lasing}

To overcome the problem of ultrafast gain decay, development of stimulated emission must occur on time scales that are shorter than those for Auger decay. One approach to enhancing the rate of stimulated emission is to increase the gain magnitude by increasing the dot density in the sample. Our estimations show that the stimulated emission rate exceeds that for the Auger recombination at NQD filling factors, $\xi$, of $\sim 1 \%$ or higher. Because of the limited NQD solubility in TOP, such densities are difficult to achieve in TOP solutions. However, they are readily achieved in solid-state NQD films (NQD solids). In particular, films made of TOPO-capped 1.3-nm NQDs have filling factors as high as ca. $20 \%$, assuming random close packing.

As expected, NQD films show stimulated emission (figure 7). We observed

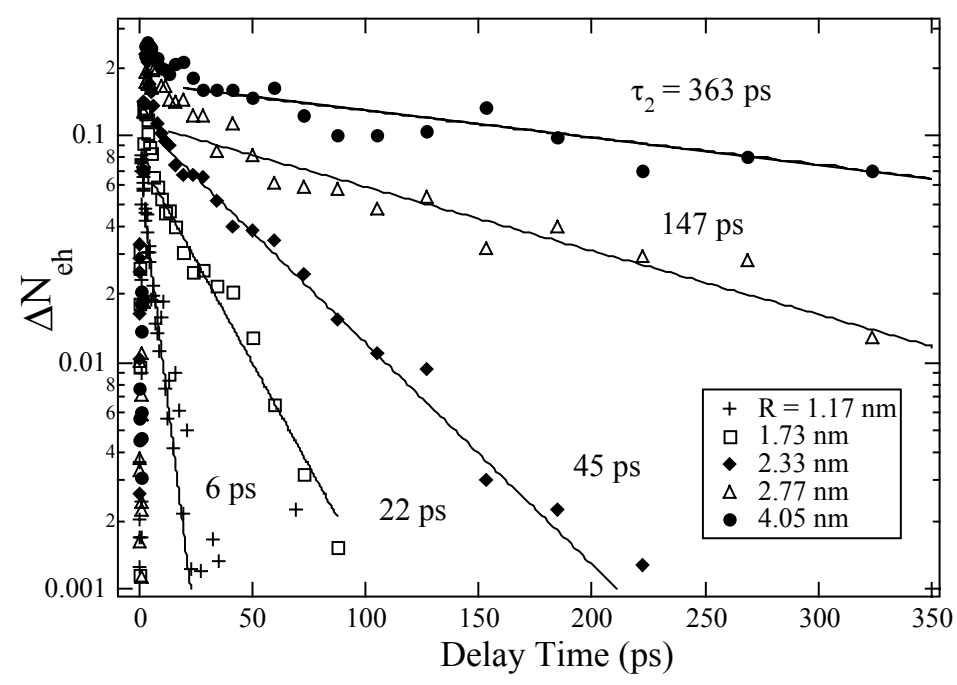

Figure 6. Size-dependent Auger rates: biexciton dynamics as a function of the NQD radius. development of stimulated emission at both room and liquid nitrogen temperatures, and the emission color was tunable by simply changing the size of the NQDs. We have also demonstrated true cavitymode lasing by depositing films on the inner walls of micro capillary tubes and promoting stimulated emission both along the length of the tube (waveguide mode) and around the inner circumference of the tube 

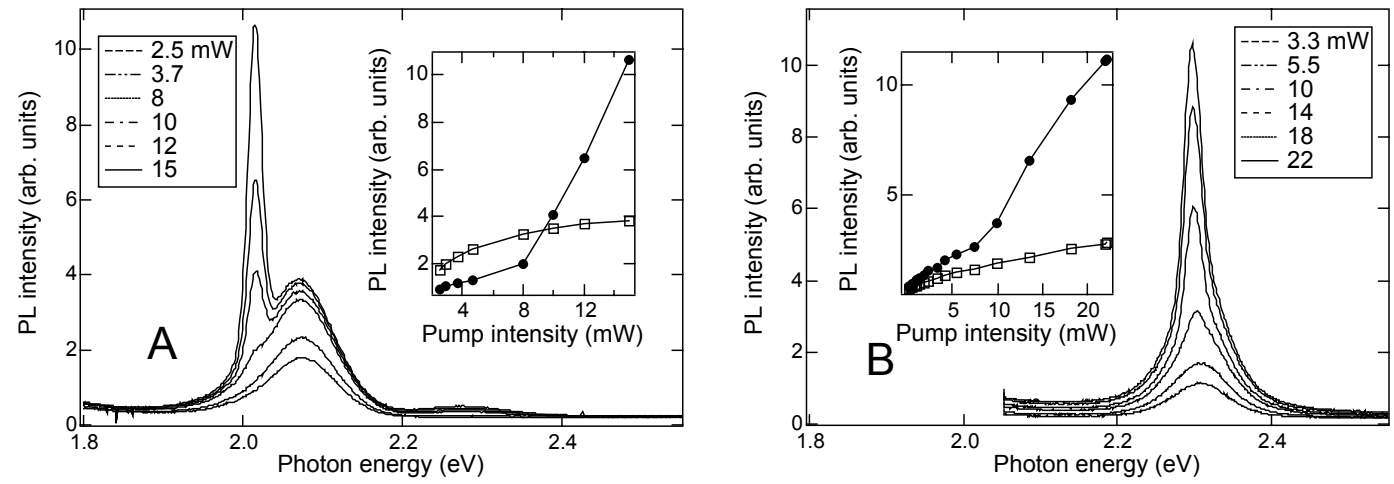

Figure 7. Development of a sharp stimulated emission band as a function of pump intensity in emission spectra of films $(T=80 \mathrm{~K})$ fabricated from TOPO-capped NQDs with $R=2.1 \mathrm{~nm}$ (A) and ZnS-capped NQDs with $R=1.35 \mathrm{~nm}(\mathrm{~B})$. The films are optically pumped perpendicular to the sample plane and the stimulated emission is detected at the edge of the films that act as optical waveguides. Insets: Superlinear intensity dependence of the stimulated emission (circles) showing a clear threshold compared to the sublinear dependence of the PL intensity outside the sharp stimulated emission peak (squares).

(whispering gallery mode) [12]. These results provide a proof-of-principle for lasing in strongly confined NQDs and should motivate the development of tunable NQD-based lasers and amplifiers operating over a broad spectral range.

\section{REFERENCES}

1. M. Asada, Y. Miyamoto, and Y. Suematsu, IEEE J. Quantum Electron. QE-22, 1915 (1986).

2. N. N. Ledentsov, et al., Semiconductors 28, 832 (1994).

3. N. Kistaedter et al., Electron. Lett. 30, 1416 (1994).

4. M. Grundman, Physica E 5, 167 (2000).

5. C. B. Murray, D. J. Norris, and M. G. Bawendi, J. Am. Chem. Soc. 115, 8706 (1993).

6. V. Klimov and D. McBranch, Opt. Lett. 23, 277 (1998).

7. V. I. Klimov, Ch. J. Schwarz, D. W. McBranch, C. A. Leatherdale, and M. G. Bawendi, Phys. Rev. B 60, R2177 (1999).

8. V. I. Klimov, A. A. Mikhailovsky, Su Xu, A. Malko, J. A. Hollingsworth, C. A. Leatherdale, H. -J. Eisler, and M. G. Bawendi, Science 290, 314 (2000).

9. M. Nirmal et al., Phys. Rev. Lett. 75, 3728 (1995).

10. V. I. Klimov, A. A. Mikhailovsky, D. W. McBranch, C. A. Leatherdale, and M. G. Bawendi, Science 287, 10117 (2000).

11. D. Chepic, A. L. Efros, A. Ekimov, M. Ivanov, V. A. Kharchenko, and I. Kudriavtsev, J. Luminescence 47, 113 (1990).

12. In progress. 